Marquette University

e-Publications@Marquette

$11-2015$

\title{
The HOMO Nodal Arrangement in Polychromophoric Molecules and Assemblies Controls the Interchromophoric Electronic Coupling
}

\author{
Marat R. Talipov \\ Marquette University, marat.talipov@marquette.edu \\ Tushar Navale \\ Marquette University \\ Rajendra Rathore \\ Marquette University, rajendra.rathore@marquette.edu
}

Follow this and additional works at: https://epublications.marquette.edu/chem_fac

Part of the Chemistry Commons

\section{Recommended Citation}

Talipov, Marat R.; Navale, Tushar; and Rathore, Rajendra, "The HOMO Nodal Arrangement in Polychromophoric Molecules and Assemblies Controls the Interchromophoric Electronic Coupling" (2015). Chemistry Faculty Research and Publications. 458.

https://epublications.marquette.edu/chem_fac/458 
Marquette University

e-Publications@Marquette

\title{
Chemistry Faculty Research and Publications/College of Arts and Sciences
}

This paper is NOT THE PUBLISHED VERSION.

Access the published version at the link in the citation below.

Angewandte Chemie International Edition, Vol. 54, No. 48 (November 23, 2015): 14468-14472. DOI.

This article is (C) Wiley permission has been granted for this version to appear in $\underline{\mathrm{e}-}$

Publications@Marquette. Wiley does not grant permission for this article to be further

copied/distributed or hosted elsewhere without the express permission from Wiley].

\section{The HOMO Nodal Arrangement in Polychromophoric Molecules and Assemblies Controls the Interchromophoric Electronic Coupling}

\author{
Dr. Marat R. Talipov \\ Department of Chemistry, Marquette University, Milwaukee, WI \\ Dr. Tushar S. Navale \\ Department of Chemistry, Marquette University, Milwaukee, WI \\ Prof. Rajendra Rathore \\ Department of Chemistry, Marquette University, Milwaukee, WI
}

\author{
Abstract \\ Triptycenes spontaneously assemble into two-dimensional networks in which long-range charge \\ transport is facilitated by the extensive electronic coupling through the triptycene framework \\ (intramolecularly) and by cofacial $\pi$-stacking (intermolecularly). While designing and synthesizing next-
}


generation triptycenes containing polyaromatic chromophores, the electronic coupling amongst the chromophores was observed to be highly dependent on the nature and position of the substituents. Herein, we demonstrate using hexaalkoxytriptycenes that the electronic coupling amongst the chromophores is switched on and off by a simple repositioning of the substituents, which alters the nodal arrangement of the HOMOs of the individual chromophores. A visual inspection of the HOMOs can thus provide a ready evaluation of the electronic coupling in polychromophoric molecules/assemblies, and will serve as an important tool for the rational design of modern chargetransport materials.

The design and synthesis of next-generation cofacially arrayed molecular assemblies is essential for achieving long-range charge transport/separation and critically needed to improve the functionality and performance of photovoltaic devices..$^{1-4}$ For example, we have recently shown that triptycenes and their radical cations assemble into two-dimensional networks where the benzenoid chromophores are electronically coupled both intramolecularly through the triptycene scaffold and intermolecularly through $\pi$-stacking (Figure 1). ${ }^{4}{ }^{6}$

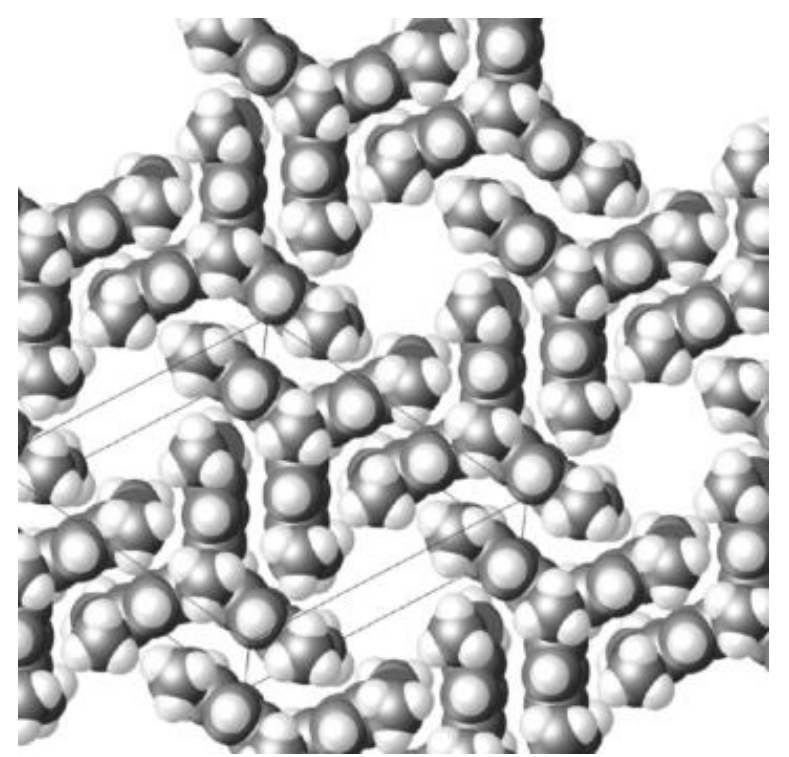

Figure 1. A cofacially arrayed 2D assembly of the hexamethoxytriptycene cation radical, determined by X-ray crystallography. ${ }^{4}, 5$

Despite the potential of the triptycene core for designing novel charge-transport materials, the effects of substituents and their positions as well as changing benzenoid rings to polyaromatic chromophores in modulating the interchromophoric coupling have not been studied. Herein, we show that the electronic coupling in the hexaalkoxytriptycenes T23 and T14 can be turned on and off by a simple repositioning of the substituents. 

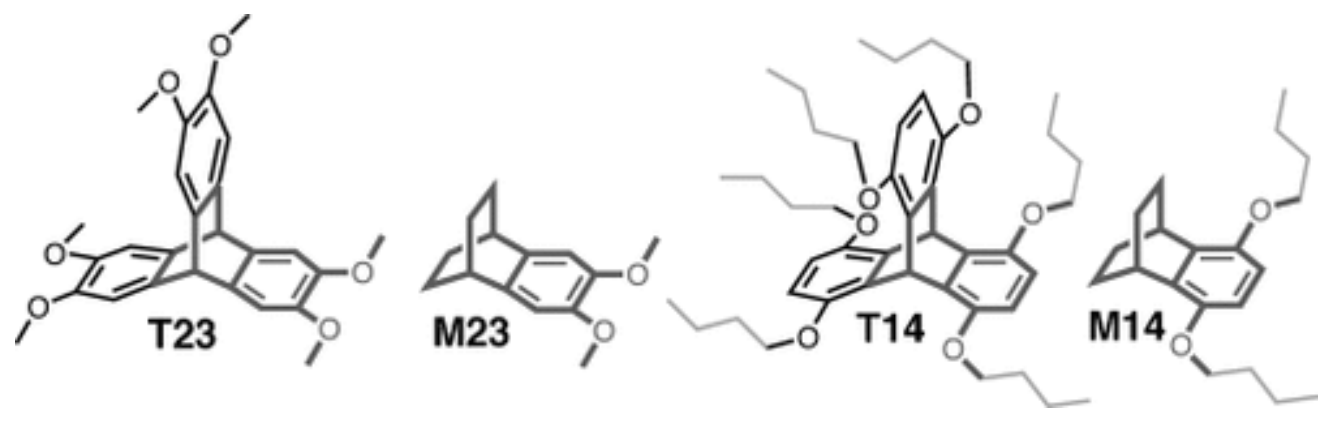

This unprecedented observation was carefully analyzed both experimentally (electrochemistry, electronic spectroscopy, and crystallography) and computationally (DFT calculations and Hückel theory), and we show that the electronic coupling can be evaluated by a simple visual inspection of the HOMOs of the neutral molecules. An easily employable method for evaluating the extent of electronic coupling in multichromophoric assemblies beyond triptycenes has thus been developed.

The synthesis of T23 and T14 and the corresponding model compounds M23 and M14 as well as their characterization by ${ }^{1} \mathrm{H} /{ }^{13} \mathrm{C}$ NMR spectroscopy and X-ray crystallography are described in the Supporting Information. ${ }^{7}{ }^{9}$ Butoxy-substituted T14 and its methoxy analogue showed similar redox potentials but they differed dramatically in their solubility. Therefore, further experimental data were collected using readily soluble $\mathbf{T} 14$.

The reversible voltammograms of T23 and T14 and the model compounds were recorded at a platinum electrode in $\mathrm{CH}_{2} \mathrm{Cl}_{2}$ and referenced to ferrocene (Figure 2 a). Interestingly, the $E_{\text {ox1 }}$ potential

of T23 (0.51 V vs. Fc/ $\left.\mathrm{Fc}^{+}\right)$was $190 \mathrm{mV}$ lower than that of T14 $(0.70 \mathrm{~V})$ whereas the $E_{\text {ox } 1}$ value of model compound M23 (0.71 V) was $70 \mathrm{mV}$ higher than that of M14 (0.64 V). ${ }^{10},{ }^{11}$ Moreover, the $E_{\text {ox1 }}$ of $\mathbf{T} 23$ was $200 \mathrm{mV}$ lower than that of model compound $\mathbf{M} 23$, whereas the $E_{\text {ox } 1}$ of T14 was 60 $\mathrm{mV}$ higher than that of model compound M14. This surprising experimental observation suggests extensive electronic coupling amongst the 2,3-dialkoxybenzene units in T23 and its absence in T14, the 1,4-dialkoxybenzene analogue. Moreover, the higher oxidation potentials of T23 and T14 (Figure 2 a) show a wide spread, which is due to the interplay between electronic coupling and Coulombic repulsion. ${ }^{12}$
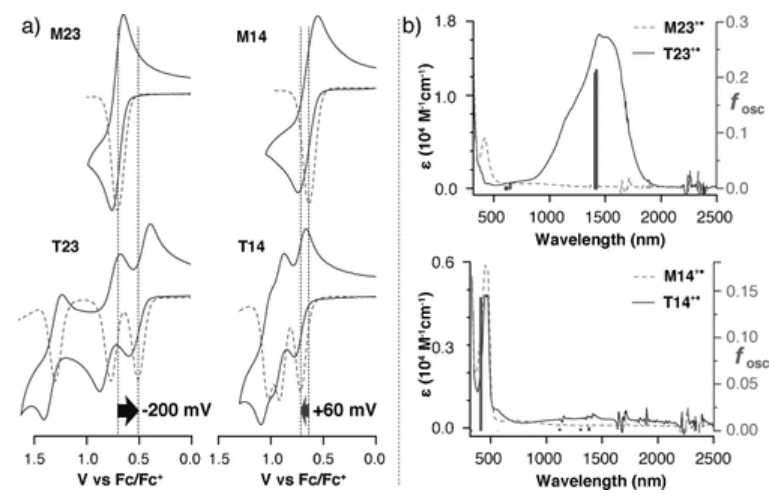

Figure 2. a) Cyclic (-) and square-wave (-- ) voltammograms of $2.5 \mathrm{mM} \mathrm{M23}(0.71 \mathrm{~V}), \mathbf{T 2 3}(0.51,0.76,1.29$ V), M14 (0.64 V), and T14 (0.70, 0.90, $1.00 \mathrm{~V})$ in $\mathrm{CH}_{2} \mathrm{Cl}_{2}\left(0.2 \mathrm{M} \mathrm{nBu}_{4} \mathrm{NPF}_{6}\right)$ at $22{ }^{\circ} \mathrm{C}, v=200 \mathrm{mV} \mathrm{s}^{-1}$. b) Absorption spectra of $\mathrm{T}^{2} 3^{+} / \mathrm{M}^{2} 3^{+}$and $\mathrm{T} 4^{+} / \mathrm{M} 4^{+}$. Thick vertical lines indicate the wavelengths and oscillator strengths of electronic excitations determined by TD-DFT calculations [B1LYP-40/6-31G(d)+PCM $\left(\mathrm{CH}_{2} \mathrm{Cl}_{2}\right)$ ]. 
Electronic coupling amongst the aromatic chromophores in cofacially arrayed assemblies also gives rise to an intervalence transition in the near-infrared (NIR) region of the electronic spectra of the corresponding radical cations (RCs). ${ }^{4},{ }^{13}-{ }^{16}$ Accordingly, the RCs of various triptycenes and model compounds were quantitatively generated by redox titration with the robust aromatic oxidants $\left[\mathrm{THE}^{+}\right]\left[\mathrm{SbCl}_{6}{ }^{-}\right]^{17}$ and $\left[\mathrm{NAP}^{+}\right]\left[\mathrm{SbCl}_{6}{ }^{-}\right]^{18}$ (THE=1,2,3,4,5,6,7,8-octahydro-9,10-dimethoxy-1,4:5,8dimethanoanthracene, NAP=1,2,3,4,7,8,9,10-octahydro-1,1,4,4,7,7,10,10-octamethylnaphthacene). The clean one-electron oxidation reactions of neutral T23, T14, M23, and M14 were confirmed by UV/Vis spectroscopic titrations, each of which showed well-defined isosbestic points and stoichiometric consumption of the oxidants (Supporting Information, Figure S1). The appearance of the electronic spectra of the various RCs $\left(\mathrm{T}_{23^{+}}{ }^{+}, \mathbf{T} 14^{+}, \mathbf{M} \mathbf{3}^{+}\right.$, and $\mathbf{M} 14^{+}$; ; see Figure $\left.\mathbf{2} \mathbf{b}\right)$ was independent of the nature of the oxidant or counteranion used, as established by their generation with other oxidants, such as $\mathrm{NO}^{+} \mathrm{SbCl}_{6}{ }^{-}, \mathrm{NO}^{+} \mathrm{BF}_{4}{ }^{-}, \mathrm{NO}^{+} \mathrm{SbF}_{6}{ }^{-}$, and $\mathrm{Et}_{3} \mathrm{O}^{+} \mathrm{SbCl}_{6}{ }^{-}{ }^{19},{ }^{20}$ Moreover, the spectra in Figure $2 \mathbf{b}$ did not change even when the RC concentration was either increased or decreased by a factor of ten, suggesting a lack of dimerization or aggregation.

As the presence of an intense NIR band for polychromophoric RCs is associated with through-space electronic coupling, ${ }^{4},{ }^{13}{ }^{16}$ the appearance of the NIR band for $\mathbf{T 2 3}^{+}$, in combination with the absence of an NIR band for monochromophoric $\mathbf{M 2 3}^{+}$, clearly indicates strong electronic coupling amongst the chromophores in $\mathbf{T 2 3}^{+}$. Unlike $\mathbf{T 2 3}^{+}, \mathbf{T 1 4}^{+}$. does not show an intense NIR band, and its spectrum is similar to that of its model compound $\mathbf{M} 1 \mathbf{4}^{+}$. (Figure $\mathbf{2}$ b). This observation suggests minimal interchromophoric coupling in $\mathrm{T14}^{+} .{ }^{7}$

To analyze the cause and effect of this unique observation of disabling/enabling the interchromophoric coupling by repositioning of the substituents, we employed the Hückel method and DFT calculations. Interestingly, a visual inspection of the HOMO of a neutral molecule readily provides a pictorial description of the spin/charge distribution in the corresponding RC. ${ }^{21}{ }^{25}$ For example, a comparison of the X-ray structures of neutral PAHs (PAH=polycyclic aromatic hydrocarbon) and their RCs shows that the regions of the HOMO bonding lobes undergo bond elongation while the anti-bonding regions undergo bond contraction (see Tables S1-S6 for representative examples).

The HOMO shapes of the neutral molecules can be rapidly determined by DFT calculations (B1LYP-40, B3LYP, CAM-B3LYP, M06-2X, and $\omega B 97 X-D$ in combination with the 6-31G(d) basis set/PCM $\left(\mathrm{CH}_{2} \mathrm{Cl}_{2}\right)$, see the Supporting Information), Hartree-Fock, or even semi-empirical (e.g., AM1, PM3) calculations. Visual inspection of the HOMOs of M23 and M14 shows that they bear striking similarity to the two degenerate HOMOs of benzene, which are referred to as the "quinoidal" (Q) and "bisallylic" (B) orbitals (Figure $\mathbf{3}$ a). The different order of the $Q$ and $B$ orbitals in $\mathbf{M} 23$ and $\mathbf{M} 14$ is due to the different positioning of the methoxy groups, as the oxygen lone pair orbitals of the ortho-methoxy groups in $\mathbf{M} 23$ have better overlap with the Q orbital than with the B orbital of $\mathbf{M} 23$. At the same time, the oxygen lone pair orbitals of the methoxy groups in M14 have better overlap with the B orbital than with the Q orbital (Figure $\mathbf{3}$ a). 

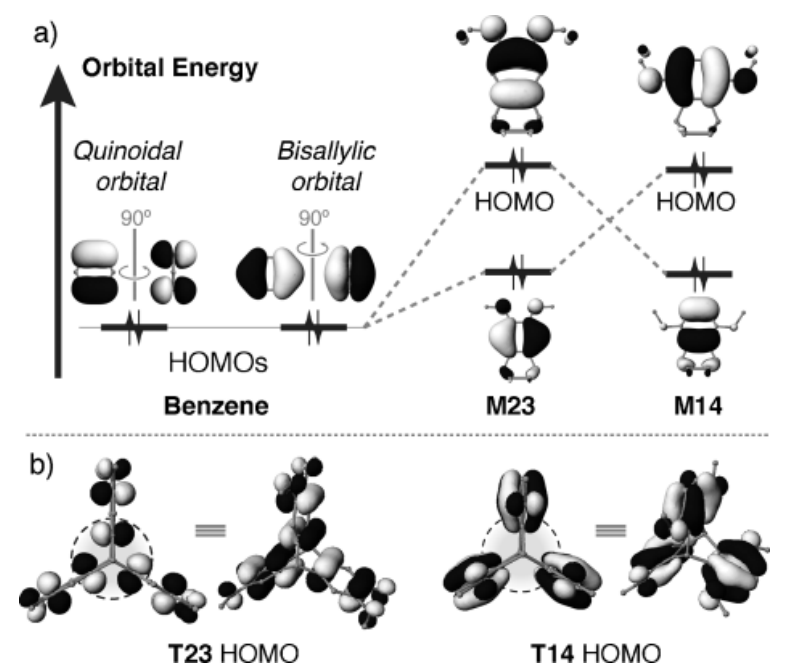

Figure 3. The HOMOs (0.03 a.u.) of a) benzene, M23, and M14, and b) T23 and T14 [B1 LYP-40/6-

$\left.31 \mathrm{G}(\mathrm{d})+\mathrm{PCM}\left(\mathrm{CH}_{2} \mathrm{Cl}_{2}\right)\right]$. The dashed circles denote the regions of interchromophoric electronic coupling in T23 and T14. See Figure S2 for the Hartree-Fock orbitals.

Interestingly, the HOMOs of T23 and T14 show that the shape of the HOMO in each chromophoric unit closely matches the shape of the HOMO in the corresponding model compounds (Figure $\mathbf{3}$ ).

Furthermore, the electronic coupling amongst the chromophores in triptycenes should critically depend on the extent of the overlap of the HOMOs of individual chromophores in the regions of interchromophoric interactions (dashed circles in Figure $\mathbf{3} \mathbf{b}$ ). Indeed, visual inspection of the shapes of the T23 and T14 HOMOs clearly suggests that the probability of finding a HOMO electron (i.e., the HOMO density) in the central part of the triptycene, which is responsible for the electronic coupling, is much higher in T23 than in T14 (Figure 3 b).

A ballpark comparison of the orbital overlap of the chromophores in triptycenes T23 and T14 can be accomplished using the Hückel method: The HOMOs of triptycenes T23 and T14 were assumed to consist of the three identical HOMOs of the monochromophoric units, which, in turn, were replaced by the HOMOs of benzene, that is, the quinoidal and bisallylic orbitals (Figure $\mathbf{4}$ a). Using this assumption, the probability of finding the HOMO electron on the "internal" aromatic carbon atoms (highlighted by dashed circles in Figure $\mathbf{3} \mathbf{b}$ ), which was calculated as the sum of the squares of the atomic orbital coefficients, was found to be three times larger in T23 than in T14, which accounts for the significantly reduced electronic coupling in T14 (Figure 4 a). ${ }^{26}$ 

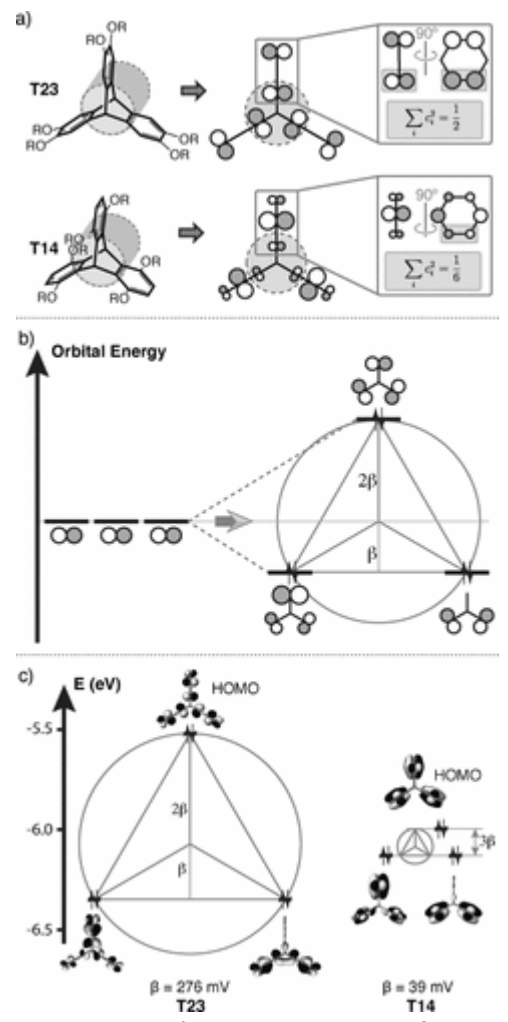

Figure 4. a) Comparison of the probability of finding an electron occupying $B$ and $Q$ orbitals on the atoms highlighted by dashed circles. b) The Frost circle corresponding to the triptycene topology with the monomer HOMOs shown as p-type orbitals for clarity. c) Scaling of the Frost circle in (b) using the calculated energies of the three HOMOs of T23 and T14. See Figure S4 for the Hartree-Fock orbitals and Table S8 for other DFT functionals.

The interaction of the HOMOs of the individual monomer units in T23 (or T14) results in Möbius topology, and therefore, a simple Hückel approach shows that the resulting filled frontier molecular orbitals will be arranged in an inverted Frost circle (Figure $\mathbf{4}$ b). Thus the HOMO of T23 (or T14) is destabilized by a value of $2 \beta$ (where $\beta$ is the electronic coupling), whereas the degenerate orbitals HOMO-1/HOMO-2 are stabilized by a value of $\beta$ with respect to the monomer HOMOs (see the Supporting Information for details).

The inverted Frost circle in Figure $\mathbf{4} \mathbf{b}$ can be quantitatively scaled by making use of the energies of the HOMO and HOMO-1/HOMO-2 of T23 obtained by DFT calculations, which then directly provides a value of $\beta($ T23) $=276 \mathrm{mV}$ (Figure $\mathbf{4} \mathrm{c}$ ). Interestingly, a similar Frost diagram for T14 (Figure $\mathbf{4} \mathrm{c}$ ) yielded a value of $\beta(\mathrm{T} 14)=39 \mathrm{mV}$, which is seven times smaller than that of T23. ${ }^{27},{ }^{28}$

Based on the dramatically different $\beta$ values for T14 and T23, the spin/charge distribution is expected to be delocalized in $\mathrm{T}^{2} 3^{+}$and more or less localized in $\mathrm{T}^{+} 4^{+}$. Indeed, the calculated spin/charge distribution in $\mathrm{T}^{2} 3^{+}$. confirms a complete delocalization of the spin/charge in $\mathrm{T}_{23}^{+}{ }^{+}$whereas in $\mathrm{T}_{14}^{+}$, it is localized on one unit (Figures $\mathbf{5}$ and S6). ${ }^{29}$ 


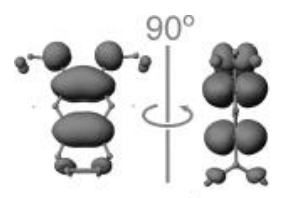

${\mathrm{M} 23^{+\bullet}}^{+\bullet}$

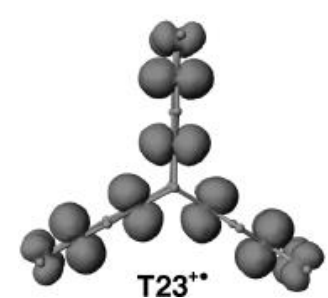

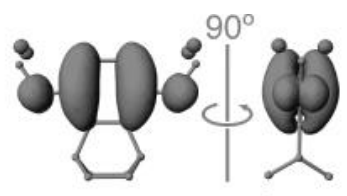

${\mathrm{M} 14^{+*}}^{+*}$

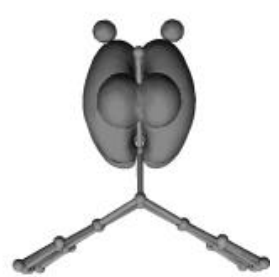

T14

Figure 5. Spin-density plots (0.001 a.u.) of $\mathbf{T 2 3}^{+}{ }^{+}, \mathrm{T14}^{++}, \mathbf{M} 23^{+}$, and $\mathbf{M} 14^{+}$[B1LYP-40/6-31G(d)+PCM(CH $\left.\left.{ }_{2} \mathrm{Cl}_{2}\right)\right]$. Only the positive component of the unpaired spin density is shown for clarity. See Figure S5 for complete spin and charge distributions.

The DFT calculations also provided the free energies of oxidation ( $\left.\Delta G_{\circ x}\right)$ for T23, T14, M23, and M14, and they were in good agreement with the experimental values (see Table S7). For example, the $\Delta G_{\text {ox }}$ value of T23 was $350 \mathrm{mV}$ lower than that of T14 whereas the $\Delta G_{\text {ox }}$ energy of M23 was only within 40 $\mathrm{mV}$ of that of M14. Importantly, the $\Delta G_{\mathrm{ox}}$ of T23 was $260 \mathrm{mV}$ lower than that of M23, whereas the $\Delta G_{\text {ox }}$ of $\mathrm{T} 14$ was $50 \mathrm{mV}$ higher than that of M14, which is in close agreement with the experimental values (see Figure $\mathbf{2}$ a). Moreover, the calculated electronic transitions (TD-DFT) in the triptycene RCs were in good agreement with those observed in the experimental electronic absorption spectra of $\mathrm{T}^{2} 3^{+}$and $\mathbf{T} 14^{+}$(Figure $\mathbf{2} \mathrm{b}$ ).

Clearly, the simple and logical approach of assessing the electronic coupling by a visual inspection of the HOMOs readily accounts for the presence/absence of electronic coupling in T23 and T14. An application of this approach to other triptycene derivatives containing PAHs as chromophores reveals a complete lack of electronic coupling in triptycenes with naphthalene, anthracene, and higher acene derivatives as chromophores because the shapes of the HOMOs render the HOMO density to be negligible in the region of the interchromophoric interaction (Figure 6). In contrast, the shapes of the HOMOs in triptycenes with biphenylene and fluorene derivatives as the interacting chromophores are indicative of strong electronic coupling because the HOMO density is high in the region of the interchromophoric interaction (Figure 6; see also Table S14 for the calculated HOMOs, the corresponding spin/charge distributions, and redox potentials). This approach is not limited to evaluating the interchromophoric electronic coupling in triptycenes but can also be applied to any cofacially arrayed polychromophoric systems as well as covalently linked $\pi$-conjugated systems (see the Supporting Information). 


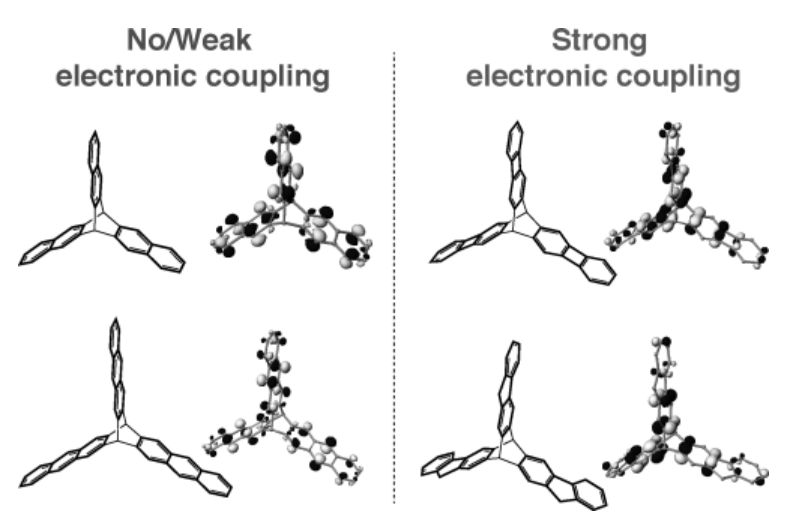

Figure 6. Evaluation of the electronic coupling amongst the interacting chromophores in representative examples based on a visual inspection of the HOMOs.

In summary, we have demonstrated that there is extensive interchromophoric electronic coupling in T23, which was gauged by the lowering of its redox potential and the appearance of an intervalence transition in the electronic spectrum of $\mathbf{T 2 3}^{+}$. compared to model compound $\mathbf{M} 23$. In contrast, the absence of electronic coupling in T14 was evident from its redox potential and the RC electronic spectrum, which were were similar to those of model compound M14. The on/off switching of the interchromophoric coupling in T23 and T14 by a simple repositioning of the substituents was easily rationalized based on the inspection of their HOMOs. This logical and intuitive approach for the quantitative evaluation of the interchromophoric electronic coupling is widely applicable and highly reminiscent of the methods used for the prediction of chemical reactivity and the regio- and stereoselectivity of a variety of organic transformations, which are based on an analysis of the frontier molecular orbitals, ${ }^{30}$ and is expected to have far-reaching implications in designing next-generation charge-transport materials for applications in photovoltaics and molecular electronics.

\section{Acknowledgements}

We thank the NSF and the NIH for financial support, and Prof. J. R. Gardiner, Prof. S. A. Reid, and Prof. Q. K. Timerghazin for helpful discussions. The calculations were performed on the high-performance computing cluster Père at Marquette University funded by the NSF awards OCI-0923037 and CBET0521602, and the Extreme Science and Engineering Discovery Environment (XSEDE) funded by NSF (TGCHE130101).

\section{References}

1 R. Rathore, S. H. Abdelwahed, I. A. Guzei, J. Am. Chem. Soc. 2003, 125, 8712-8713.

2 M. Shibahara, M. Watanabe, T. Iwanaga, K. Ideta, T. Shinmyozu, J. Org. Chem. 2007, 72, 2865- 2877.

3 M. R. Wasielewski, Acc. Chem. Res. 2009, 42, 1910- 1921.

4 T. S. Navale, K. Thakur, V. S. Vyas, S. H. Wadumethrige, R. Shukla, S. V. Lindeman, R. Rathore, Langmuir 2012, 28, 71-83.

5 V. J. Chebny, T. S. Navale, R. Shukla, S. V. Lindeman, R. Rathore, Org. Lett. 2009, 11, 2253- 2256. 6 T. Kobayashi, T. Kubota, K. Ezumi, J. Am. Chem. Soc. 1983, 105, 2172- 2174.

7 H. Quast, H. L. Fuchsbauer, Chem. Ber. 1986, 119, 1016- 1038.

8 B. S. Ghanem, M. Hashem, K. D. Harris, K. J. Msayib, M. Xu, P. M. Budd, N. Chaukura, D. Book, S. Tedds, A. Walton, Macromolecules 2010, 43, 5287- 5294. 
9 T. S. Navale, R. Rathore, Synthesis 2012, 805-809.

10 R. Rathore, J. K. Kochi, J. Org. Chem. 1995, 60, 4399- 4411.

11The similarity of the oxidation potentials of 1,2- and 1,4-dimethoxysubstituted arenes is further corroborated by a comparison of the redox potentials of 4,5-dimethyl-1,2-dimethoxybenzene (0.77 V vs. Fc/Fc ${ }^{+}$) and 2,5-dimethyl-1,4-dimethoxybenzene (0.67 V); see: V. J. Chebny, R. Shukla, S. V. Lindeman, R. Rathore, Org. Lett. 2009, 11, 1939.

12 A discussion of the second and third oxidation potentials of T23 and T14 is included in the Supporting Information.

13 J. K. Kochi, R. Rathore, P. L. Magueres, J. Org. Chem. 2000, 65, 6826-6836.

14 B. Badger, B. Brocklehurst, Nature 1968, 219, 263- 263.

15 B. Badger, B. Brocklehurst, Trans Faraday. Soc. 1969, 65, 2582- 2587.

16 B. Badger, B. Brocklehurst, Trans Faraday. Soc. 1970, 66, 2939- 2947.

17 R. Rathore, C. L. Burns, M. I. Deselnicu, Org. Synth. 2005, 1-9.

18 R. Rathore, C. L. Burns, M. I. Deselnicu, Org. Lett. 2001, 3, 2887- 2890.

19 R. Rathore, A. S. Kumar, S. V. Lindeman, J. K. Kochi, J. Org. Chem. 1998, 63, 5847- 5856. 20 See Ref. [13].

21 R. Shukla, S. H. Wadumethrige, S. V. Lindeman, R. Rathore, Org. Lett. 2008, 10, 3587- 3590.

22 M. Banerjee, V. S. Vyas, S. V. Lindeman, R. Rathore, Chem. Commun. 2008, 1889- 1891.

23 T. S. Navale, L. Zhai, S. V. Lindeman, R. Rathore, Chem. Commun. 2009, 2857- 2859.

24 See Ref. [11].

25 Note, however, that in extensively delocalized $\pi$-conjugated systems, for example, oligo- and polypara-phenylenes, the charge/hole distribution shows only limited delocalization whereas the HOMO is delocalized over the entire molecule; see, for example: M. R. Talipov, A. Boddeda, Q. K. Timerghazin, R. Rathore, J. Phys. Chem. C 2014, 118, 21400- 21408.

26 Importantly, this difference means that the overlap of the HOMOs of the monochromophoric units is reduced by a factor of three in T14 compared with that of T23. As the electronic coupling integral between two orbitals is often proportional to the overlap integral (e.g., such a linear relationship is used in the extended Hückel method), the electronic coupling amongst chromophores in triptycenes can also be expected to be three times smaller in T14 than in T23.

27 Moreover, the shape and the nodal arrangement of the DFT orbitals of T23/T14 in Figure $3 \mathrm{c}$ showed a striking similarity of the per-chromophore contribution in each of these molecular orbitals when compared with the molecular orbitals obtained by the Hückel approach (Figure $3 \mathrm{~b})$.

28 The use of other DFT methods and Hartree-Fock produced essentially the same ratio, $\beta($ T23)/ $\beta($ T14) $\approx 7-9 ;$ see Table S8.

29 It has been established by X-ray crystallography that in $\mathrm{T}^{2} 3^{+}$and its close analogue, the single positive charge is delocalized over all three benzenoid rings; see Tables S9-S13.

30 E. V. Anslyn, D. A. Dougherty, Modern physical organic chemistry, University Science, Sausalito, CA, 2006. 